\title{
ANÁLISIS DE FACTORES INTERVINIENTES RELACIONADOS AL MODELO DE UNIVERSIDADES EMPRENDEDORAS EN SUDAMÉRICA
}

\author{
Anselmo Aguilera Vargasa y Alfonso Paredes Aguirre*b \\ ${ }^{a}$ Facultad de Ingeniería y Negocios - Universidad Adventista de \\ Chile, Chile \\ ${ }^{b}$ Escuela de Posgrado - Universidad Peruana Unión, Lima, Perú \\ Recibido: 10 de febrero de 2017 Aceptado: 05 de marzo de de 2017
}

\begin{abstract}
Resumen
Esta investigación analiza los factores de desarrollo externos, de organización interna y mecanismos de apoyo al emprendimiento que traen como resultado una universidad emprendedora en instituciones Sudamericanas. Es de tipo cuantitativo, correlacional y de carácter explicativo con diseño de variables que busca conocer en qué medida éstas pueden ser predictoras de un modelo de universidades emprendedoras. La muestra correspondió a 65 universidades pertenecientes a 8 países sudamericanos con un carácter no probabilístico, cuyos datos fueron recolectados por encuestas online por los directores de ITT de las universidades seleccionadas. El modelo de Covin \& Slevin, (1991) y adaptado para universidades por MarkuerkiagaArritola, (2014) fue el utilizado en el estudio y consta de tres variables independientes que influyen sobre una variable dependiente calculándose el coeficiente de determinación de las variables para el contraste de las hipótesis, cuyos hallazgos muestran que se relacionan positiva y significativamente con los resultados obtenidos en universidades que implementan un modelo de gestión tipo universidad emprendedora. También se hizo un análisis SEM que permitió conocer el componente estructural relacionado con el tamaño de la muestra que fue significativo para el peso de las variables independientes sobre la dependiente, así como la covarianza entre ellas, así como los coeficientes de variables FAE y MAE que son directamente proporcionales con relación a la variable RUE. Finalmente, la dependencia geográfica, el estatus de dependencia y la pertenencia de un parque tecnológico mostraron diferencia estadística significativa para la gran mayoría de las dimensiones de las variables.
\end{abstract}

Palabras Clave: Universidades emprendedoras, factores ambientales externos, factores organizativos internos, mecanismos de apoyo al emprendimiento, Resultado de universidad emprendedora

\section{Abstract}

This research analyzes the factors of external development, internal organization and support mechanisms for entrepreneurship that result in an entrepreneurial university in South American institutions. It is of a quantitative, correlational and explanatory type with a design of variables that seeks to know to what extent these can be predictors of a model of entrepreneurial universities. The sample corresponded to 65 universities belonging to 8 South American countries with a non-probabilistic character, whose data were collected by online surveys by the ITT directors of the selected universities. The model by Covin \& Slevin, (1991) and adapted for universities by Markuerkiaga-Arritola, (2014) was the one used in the study and consists of three independent variables that influence a dependent

\footnotetext{
*Email:alfonso.paredes@upeu.edu.pe
} 
variable, calculating the coefficient of determination of the variables for the contrast of the hypotheses, whose findings show that they are positively and significantly related to the results obtained in universities that implement an entrepreneur-type university management model. An SEM analysis was also made that allowed us to know the structural component related to the sample size that was significant for the weight of the independent variables on the dependent as well as the covariance between them, as well as the coefficients of variables AED and MAE that are directly proportional to the variable RUE. Finally, the geographical dependence, the dependency status and the belonging of a technology park showed a significant statistical difference for the great majority of the dimensions of the variables.

Keywords: Entrepreneurial Universities, External Environmental Factors, Internal Organizational Factors, Support Mechanisms for Entrepreneurship, Result of an Entrepreneurial University, Covin \& Slevin Model (1991)

\section{Introducción}

El sistema universitario en América Latina y El Caribe se ha diversificado mucho durante los últimos 25 años, y las universidades tradicionales han visto cómo el crecimiento de instituciones privadas o pertenecientes a corporaciones sin fines de lucro han comenzado a ganar un espacio importante dentro del mercado universitario (Rolando, Salamanca \& Aliaga 2010; Cancino \& Schmal 2014) permitiendo que el universo de estudiantes en educación superior aumente desde 521.880 en el año 2002 hasta 1.127 .200 en el 2012 como es en el caso de Chile (OCDE, 2013a) y desde 2.500 alumnos a 3.700 alumnos por cada 100.000 habitantes en América Latina y el Caribe lo que significa un aumento de más del 40\% durante la última década (Unesco, 2014).

Este crecimiento del sector ha permitido un aumento significativo de la oferta de profesionales que la sociedad necesitaba y está siendo un componente importante en la construcción y consolidación de la clase media chilena (OCDE, 2013b) así como un fuerte motor de desarrollo en los diferentes países de América Latina que han decidido fortalecer e invertir en la educación superior (Moreno-Brid \& Ruiz-Nápoles 2008). 
Durante los últimos 4 años, una sombra de este crecimiento ha comenzado a ser la preocupación sobre la calidad de las instituciones y el proyecto que están desarrollando en el mercado, lo que ha traído aparejado una gran preocupación de los estudiantes y de la comunidad en general. Prueba de ello han sido los conflictos públicos de algunas casas de estudios vinculadas a los conceptos de calidad, gestión financiera, liderazgo administrativo lo que ha llevado a que algunas de ellas hayan visto reducir sus años de acreditación institucional, otras a perder la certificación de acreditación, e incluso algunas a ser cerradas (CNDE, 2014).

Algunos teóricos consideran los modelos de gestión como reduccionistas ya que se enfocan solamente a los aspectos financieros o son ejecutados bajo ideologías doctrinarias sobre lo que se estima que debe ser "hacer y dirigir" universidades (Lolas, 2006), esto podría significar que éstas visiones terminen obedeciendo a sus directorios, dueños, o corporaciones que las dirigen. Por otro lado está el hecho que el poder del ejercicio de la gestión puede determinar el rumbo de la institución y la burocracia profesional puede terminar en un desarrollo vegetativo con una estructura centrada mayormente en su núcleo operativo gerencial (Thompson, Strickland, Gamble \& Peteraf, 2012).

La educación superior ha pasado desde los modelos de gestión tipo "torre de marfil" (Coughlan \& Perryman, 2013) hasta los modelos modernos que desarrollan una gestión pensada en el entorno, apoyándose en las tareas, metas, prioridades y delegación de poder. Esta clásica forma de gestión da cuenta de acciones autoritarias basadas en el imperio del conocimiento, adoptando este mismo imperio características 
Revista de Investigación Universitaria | Volumen 6 - Número 2, Julio - Diciembre, 2017 | DOI :

tipo "aristocráticas", donde los sabios están a la cabeza y el resto obedece (Lolas, 2006).

Ya sea porque se haya adscrito a un modelo de gestión específico o no, existen en la práctica, administraciones universitarias tipo empresariales con el propósito de generar lucro para sus accionistas como lo son las existentes en Estados Unidos, Perú, Brasil o Costa Rica (Bernasconi, 2013) o aquellas que están cambiando el foco de la educación erudita a centros productores de ingresos para el estado como ocurre con algunas universidades en Inglaterra (Callincos, 2006). Estos tipos de modelos de gestión universitaria podría llevarlas a focalizar sus intereses económicos en primer lugar por sobre el producto social que son los profesionales de calidad, entendiendo que su sentido es generar nuevas ideas y con ellas satisfacer a consumidores (Sanchez \& Ríos, 2011). Un ejemplo de lo anterior se ve representado en lo que ha sucedido en Chile en el año 2012 con el caso de la Universidad del Mar (CNDE, 2012), cuyos intereses estaban más en la generación de ganancias y lucro para sus accionistas que de la calidad misma de la educación.

Existe una aceptación transversal en el aporte académico, administrativo y formativo que hacen las universidades hacia la sociedad y el entorno (Huggins \& Johnston, 2009) así como la nueva visión de interacción en una triple hélice entre las universidades, el estado y la industria, lo que hace que se generen nuevos espacios de desarrollo para el conocimiento (Etzkowitz, 2003a). En este nuevo escenario, los procesos de gestión administrativa en las universidades emprendedoras, comienzan a adquirir características especiales, por lo que esta investigación analizará la pregunta ¿Cuáles son los 
Revista de Investigación Universitaria | Volumen 6 - Número 2, Julio - Diciembre, 2017 | DOI :

factores que se relacionan significativamente con el modelo de universidades emprendedoras en Sudamérica?

\section{Marco teórico}

El término de Universidades Emprendedoras (UE), que según Slaughter (Valera-loza, 2010) puede ser rastreado desde la década de los 70" bajo el término de "capitalismo académico", fue acuñado por Etzkowitz, (1983) para referirse a los mecanismos con que las universidades demostraron ser fundamentales para el desarrollo de la región en la cual están insertas. Posteriormente sería Clark, (1998), quien en sus investigaciones categorizaría este tipo de instituciones en aquellas que redefinen su propia estructura para transformarse en agentes de empresa.

Existen autores que han intentado proponer una clasificación taxonómica que incluya los componentes vinculantes de este tipo de institución. Un ejemplo de ellos es la propuesta de Grimaldi que considera tres niveles distintivos dentro de las UE tales como: las características locales, la estructura institucional y el tipo de gobierno institucional (Grimaldi et al., 2011). Otros autores ponen énfasis en los procesos de la generación del conocimiento y la educación como elementos clasificatorios de emprendimiento, quienes propone que una de las formas para mantener el espíritu de emprendimiento académico es mediante la comercialización del conocimiento y las investigaciones (Klofsten \& Jones-Evans, 2000; Roessner, Bond, Okubo, \& Planting, 2013) permitiendo que sea precisamente la investigación y la transferencia tecnológica el elemento conector con la sociedad (Mowery, Sampat \& Ziedonis, 2002).

Estos procesos de generación del conocimiento también están relacionado a la enseñanza y la construcción de competencias de emprendimiento como vínculo que genere la conexión hacia 
Revista de Investigación Universitaria | Volumen 6 - Número 2, Julio - Diciembre, 2017 | DOI :

la tercera misión, característica propia de las universidades modernas (Altmann \& Ebersberger, 2013) o como también diría Urbano, el emprendimiento en el sistema de educación superior es un productor de conocimiento así como un diseminador de la institución (Guerrero \& Urbano, 2012). La Tabla 1 identifica algunas de las características distintivas de las universidades emprendedoras.

Tabla 1

Características de una Universidad Emprendedora

\begin{tabular}{ll}
\hline $\mathbf{N}^{\circ}$ & $\begin{array}{l}\text { Factores descriptivos de universidades } \\
\text { emprendedoras }\end{array}$ \\
\hline 1 & Educación en emprendimiento \\
2 & Creación de empresas \\
3 & Transferencia tecnológica \\
4 & Impacto socioeconómico en la zona \\
5 & Generación de ingresos \\
6 & Cambios estructura interna \\
\hline
\end{tabular}

Desde sus orígenes, las instituciones que generan emprendimiento han sido un aporte de desarrollo significativo para su propio entorno y han logrado desarrollar un rol trascendente no solo en la creación y la diseminación del conocimiento, sino también en aquellas características que las hacen un motor de desarrollo por su vínculo con la empresa y el medio ambiente (Pinto, Fernandez-Esquinas, \& Uyarra, 2013). Estas características han sido estudiadas por distintos investigadores a través de los años quienes han propuesto aportes conceptuales adicionales a los anteriores lo que ha enriquecido aún más la caracterización y comprensión de dichas instituciones. Algunas de estas características están descritas en la Tabla 2 .

Tabla 2

Revisión de las definiciones y funciones universidades emprendedoras

Definiciones $y$ funciones sobre UE 


\begin{tabular}{|c|c|}
\hline $\begin{array}{l}\text { (Chrisman, } \\
\text { 1995) }\end{array}$ & $\begin{array}{c}\text { Consiste en la creación de nuevos negocios por } \\
\text { docentes, técnicos y estudiantes. }\end{array}$ \\
\hline Röpke, (1998) & $\begin{array}{l}\text { Significan tres cosas: (1) la institución se } \\
\text { convierte emprendedora; (2) la comunidad } \\
\text { universitaria se transforma en emprendedores (3) } \\
\text { la interacción de la universidad con el medio } \\
\text { ambiente, el acoplamiento estructural entre la } \\
\text { universidad y la región con el emprendimiento. }\end{array}$ \\
\hline Clark, (1998) & $\begin{array}{l}\text { Busca innovar en la forma de trabajar, provocar } \\
\text { un cambio en el carácter de la organización y } \\
\text { convertirse en universidades líderes que sean } \\
\text { actores importantes en sus propios entornos. }\end{array}$ \\
\hline Kirby, (2006) & $\begin{array}{l}\text { ".. Tiene la capacidad de innovar, reconocer y } \\
\text { crea oportunidades, trabaja en equipo, toma } \\
\text { riesgos y responde a los desafíos". }\end{array}$ \\
\hline $\begin{array}{l}\text { Etzkowitz, } \\
(2003 b)\end{array}$ & $\begin{array}{c}\text { ". Capacita a estudiantes y los envía al mundo } \\
\text { y proporciona estructuras de apoyo para los } \\
\text { profesores y estudiantes para iniciar nuevas } \\
\text { empresas". }\end{array}$ \\
\hline $\begin{array}{l}\text { Tuunainen, } \\
(2005)\end{array}$ & $\begin{array}{l}\text { "Tipo de institución que está evolucionando por } \\
\text { la interacción entre la universidad, la } \\
\text { industria y el gobierno y que integra el } \\
\text { desarrollo económico en la función académica, } \\
\text { por medio de la enseñanza y la investigación". }\end{array}$ \\
\hline $\begin{array}{l}\text { Blenker, } \\
\text { Dreisler \& } \\
\text { Kjeldsen, } \\
(2006)\end{array}$ & $\begin{array}{c}\text { "... es una institución que proporciona la base } \\
\text { para el crecimiento y el desarrollo regional y } \\
\text { nacional a través de la cooperación estrecha e } \\
\text { intensa con su entorno". }\end{array}$ \\
\hline $\begin{array}{l}\text { Benneworth, } \\
(2007)\end{array}$ & $\begin{array}{c}\text { "... proporciona, a través de actividades de } \\
\text { transferencia tecnológica, nuevos conocimientos } \\
\text { tecnológicos demandados por las empresas para el } \\
\text { mercado mundial". }\end{array}$ \\
\hline $\begin{array}{l}\text { Cargill, } \\
(2007)\end{array}$ & $\begin{array}{c}\text { "... es la antítesis del aprendizaje tradicional } \\
\text { de la 'torre de marfil'... contribuyen al } \\
\text { desarrollo económico mediante la creación de } \\
\text { nuevas oportunidades y el apoyo para la creación } \\
\text { de nuevas empresas". }\end{array}$ \\
\hline
\end{tabular}




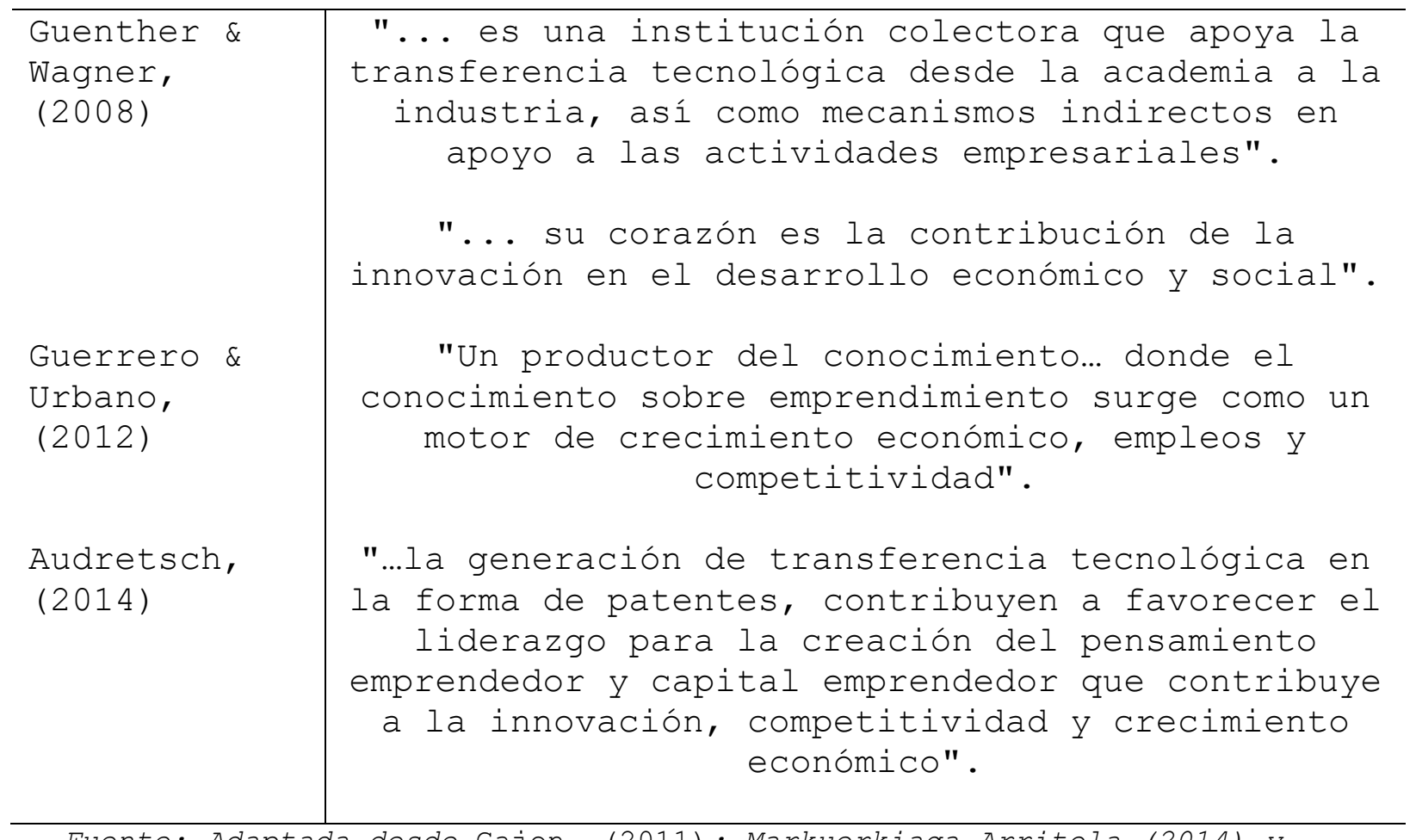

Fuente: Adaptada desde Gajon, (2011); Markuerkiaga-Arritola, (2014) y complementada.

De las declaraciones anteriormente expuestas se puede concluir que no hay una definición integradora única sobre UE, sin embargo, es evidente que, por sus múltiples características, es más que una sola definición (MarkuerkiagaArritola, 2014). Los conceptos distintivos que más destacan son la capacidad de la institución para adaptarse a los cambios, el tipo de directrices y reglamentación interna, la promoción de una cultura de emprendimiento en estudiantes, docentes y directivos, una vocación de servicio hacia el desarrollo de la comunidad y la capacidad de innovar, entre otros elementos.

Estas nuevas conceptualizaciones representan el cambio de cosmovisión que se ha desarrollado a través del tiempo de lo que es la misión de una universidad. Estos nuevos cambios económicos, tecnológicos y políticos han generado una segunda revolución académica (Etzkowitz, (2004, 2003a); Etzkowitz \& 

DOI :

Ranga, (2012)) que ha impulsado a las instituciones a replantearse su propio rol. Figura 1 nuestra precisamente esta evolución de cosmovisión.

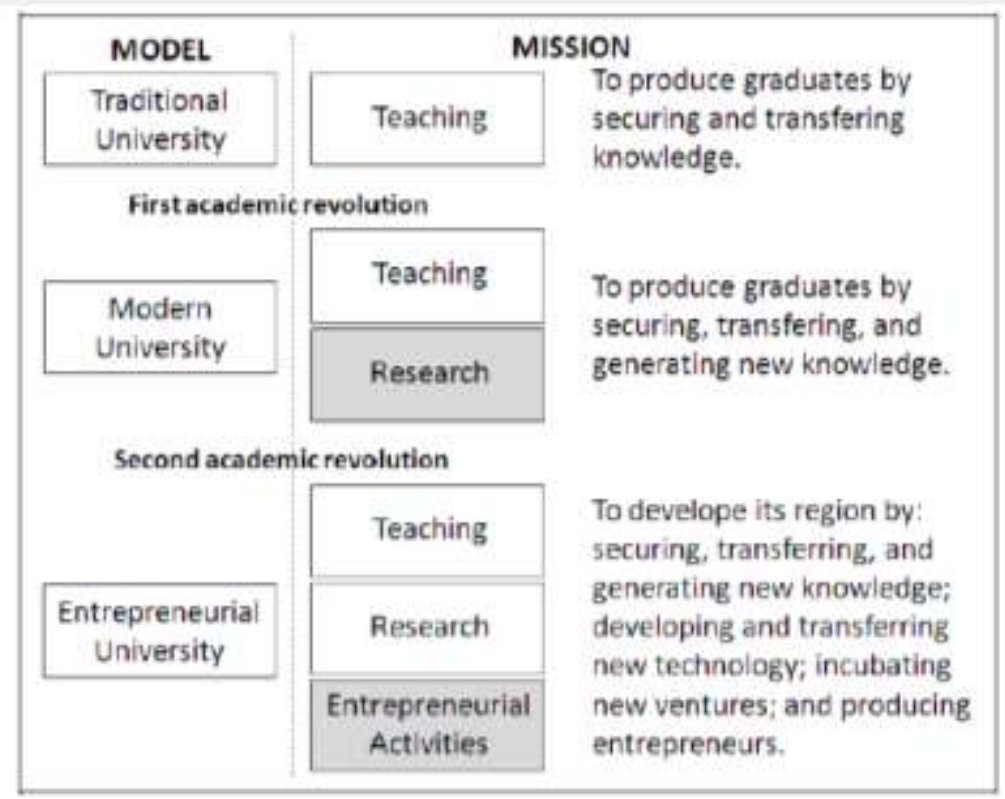

Universitarias

Figura 1 Revoluciones Académicas

Fuente: (Gajon, 2011)

Por otro lado, el marco teórico de los modelos de Emprendimiento Corporativo muestra características que se intersectan con los modelos de UE lo que permite determinar que las fortalezas internas que pudieran tener las universidades o instituciones determinarán la forma como impactan en el entorno y a su vez este mismo entorno mirará las instituciones como medios para lograr el desarrollo.

Las definiciones de emprendimiento corporativo tienen distintas miradas desde la perspectiva del origen. Existen dos cosmovisiones que pretenden caracterizar el comportamiento de innovación de los empleados, estos son el "emprendimiento corporativo" y el "intraemprendimiento" (Amo, 2006), siendo el primero el que se desarrolla como estrategia de la organización para aumentar su ventaja competitiva como una combinación de 
Revista de Investigación Universitaria | Volumen 6 - Número 2, Julio - Diciembre, 2017 | DOI :

actividades formales e informales (Belousova, Bailly \& Basso, 2010) y el segundo el iniciado desde abajo hacia arriba por un empleado para satisfacer sus intereses.

Esta investigación basó su estudio en el modelo de Covin \& Slevin, (1991) que describe conceptualmente de comportamiento emprendedor a nivel de la organización, describiendo las ventajas de adoptar dichas conductas en la construcción del espíritu de emprendimiento.

Para los autores, una postura emprendedora se ve reflejada en al menos tres tipos de comportamiento organizacional, como lo son la toma de riesgos por la alta dirección relacionadas con las decisiones de inversión y acciones estratégicas, la amplitud y frecuencia de la innovación de productos/tendencias relacionadas con el liderazgo y la forma como la empresa es capaz de competir proactivamente con los rivales de la industria (Covin \& Slevin, 1991), por lo que su concepción de emprendimiento refleja precisamente esta visión. La postura emprendedora de la institución se ve influenciada directamente por la forma como estas tres variables interactúan, generando innovación, proactividad y riesgo en la inversión, asumiendo proyectos con posibilidad de altos rendimientos y buscando nuevas oportunidades (Crow, 2008). La Figura 2 muestra el modelo de Covin \& Slevin y la interacción de estas variables. 


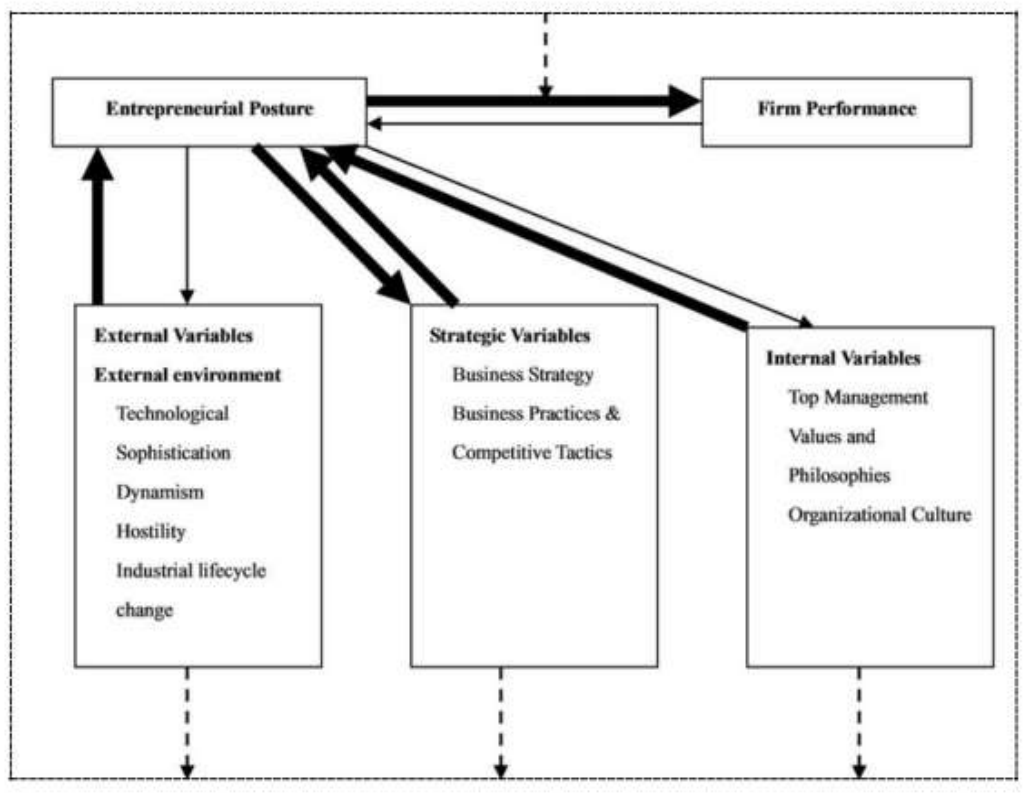

Figura 2 Modelo de Covin \& Slevin

Desde una mirada práctica las instituciones de educación superior han avanzado hacia el desarrollo de actividades de emprendimiento académico directo e indirecto manifestándose en actividades de spin-off académico (Steffensen, Rogers \& Speakman, 2000), patentamiento y licenciamiento (Powers \& Macdougall, 2005) para las primeras e investigación financiada por la industria y el desarrollo de I+D (Dooley \& Kirk, 2007) para las segundas.

Los estudios bibliográficos han intentado conceptualizar el aporte de este tipo de modelo de gestión y los modelos teóricos han intentado clasificar las instituciones según sus énfasis de desarrollo interno o focalizado hacia el medio. Según Markuerkiaga-Arritola, (2014) son pocos los estudios que han analizado el concepto de UE de forma empírica y que hayan construido sus modelos basados en diferentes factores, generando escalas de medición del rendimiento y estableciendo indicadores (Guerrero \& Urbano, 2010; Oberman-Peterka, 2011), 
Revista de Investigación Universitaria | Volumen 6 - Número 2, Julio - Diciembre, 2017 | DOI:

lo que dimensiona la importancia de profundizar en este tipo de investigaciones sobre todo en universidades sudamericanas ya que en dichos países se están desarrollando distintas modificaciones legales que apuntan al fortalecimiento de la calidad en educación superior por lo que necesitamos evaluar y buscar mejores modelos de gestión universitaria que puedan ayudar a elevar los indicadores y resultados.

De la información que los distintos autores aportan sobre las definiciones conceptuales, es posible identificar las características que estas instituciones académicas tienen, las cuales se ven caracterizadas por:

a. El fomento permanente de una cultura emprendedora en todos los niveles en que la institución desarrolla su accionar, impulsando cambios desde los estudiantes, académicos y hasta la estructura institucional (Gibb \& Hannon, 2005; Meyer, 2011).

b. Desarrolla una visión multifactorial acerca del ingreso financiero institucional favoreciendo ingresos que no son directos a la función de la docencia (Clark, 2004).

c. Visualizan el medio en el cual están insertos y donde pueden ser funcionales como para reestablecer su estructura, los procesos de gestión sus objetivos principales y su misión (Bratianu \& Stanciu, 2010; Meyer, 2011 ).

d. Por causa de su fuerte relación con el entorno, termina influyendo en el desarrollo económico y social de la comunidad directa (Etzkowitz, 2003b; Bratianu \& Stanciu, 2010)

De igual forma, varios autores (H. Etzkowitz, 2004; Guerrero, Urbano \& Kirby, 2006; Peterka, 2007; Mavi, 2014) han tratado de identificar las caracteristicas que llevan al desarrollo de una EU a través de otras variables, la mayoría de ellos basaron sus marcos teóricos en factores completamente diferentes, sin embargo los factores mayoritariamente mencionados corresponden a la misión y la estrategia, la 
educación hacia el emprendimiento, la estructura de gobierno, los métodos de enseñanza y el ambiente externo (Markuerkiaga et all. 2014).

\section{Diseño metodológico}

Esta investigación se clasifica del tipo cuantitativa, correlacional y explicativa, pues encontrar la correlación entre dos o más variables permite predecir el valor que podrán tener los sujetos de estudios sabiendo el valor que tienen en la otra variable (Sampieri et al., 2014); es otras palabras, nos permitirá conocer en qué medida las variables pueden ser predictores de un modelo de EU.

La construcción filosófica del modelo de esta investigación estableció el desarrollo del análisis deductivo ya que permite que, de establecerse las premisas como verdaderas, las conclusiones deberán ser también ciertas siguiendo el razonamiento del contraste de una hipótesis y que permita llegar a una conclusión lógica y específica (Gray, 2014).

La investigación centró su análisis en la intencionalidad de las universidades seleccionadas para el estudio en desarrollar competencias de gestión administrativas según las características propuestas por el modelo de Covin \& Slevin, (1991) y aplicado en gestión de universidades tipificadas como emprendedoras (Guerrero, Urbano, Cunningham, \& Organ, 2014) bajo el diseño de la Figura 3.

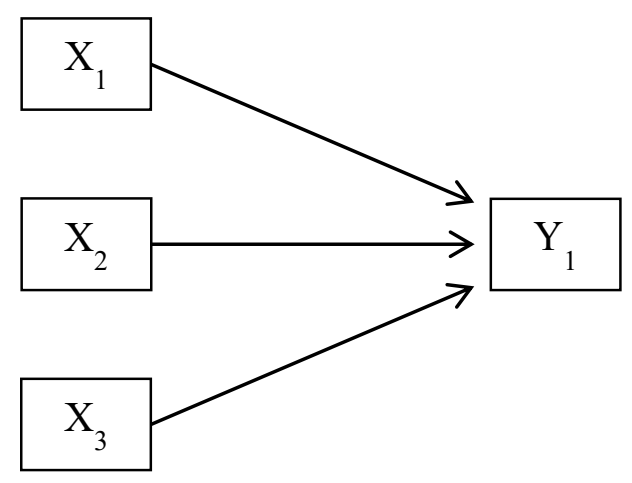
$\mathrm{X}_{1}$ : Variable de factores ambientales externos
$\mathrm{X}_{2}$ : Variable de factores organizativos internos
$\mathrm{X}_{3}$ : Variable Mecanismo de Apoyo al Emprendimiento
$\mathrm{Y}_{1}$ : Variable Resultados como Universidad Emprendedora

Figura 3: Diseño conceptual del modelo de Covin \& Slevin para variables indepenzientes $y$ dependiente 
El instrumento aplicado con sus 109 preguntas, analizó 16 áreas que representan las variables y dimensiones que están relacionadas con el emprendimiento universitario y el resultado de las comparaciones de cada una de las variables se pudo identificar cuan adaptativa estaba una institución al modelo propuesto y cuan adaptativa estaba en comparación con las otras instituciones.

El análisis de fiabilidad por concepto del Alfa de Cronbach (Tabla 3) para todo el instrumento fue de 0.959. La medida para cada una de las 22 dimensiones presentó valores superiores a 0.955 con valores de correlación elemento total corregida superiores a 0.630 con la sola excepción de la dimensión "educación continua" con 0.496 pero superior a 0.40 como exigencia mínima.

Tabla 3

Resultado calculo Alfa de Cronbach para el instrumento

\section{Estadísticos de fiabilidad}

Alfa de Cronbach

, 959
$\mathrm{N}$ de elementos

23

El desarrollo de la investigación correspondió al año 2017 e incluyó a 8 países sudamericanos de los cuales fueron seleccionadas instituciones de educación superior públicas y privadas. Los países involucrados corresponden a: Argentina, Bolivia, Brasil, Chile, Ecuador, Paraguay, Perú y Uruguay. La Tabla 4 muestra la población y muestra por países.

Tabla 4

Universo y muestra de universidades por países

\begin{tabular}{lcc}
\hline & Total de universidades participantes & \\
\hline País & $\mathrm{N}$ & $\mathrm{n}$ \\
Argentina & 26 & 9 \\
Bolivia & 7 & 3 \\
Brasil & 23 & 18 \\
\hline
\end{tabular}


Revista de Investigación Universitaria | Volumen 6 - Número 2, Julio - Diciembre, 2017 | DOI :

\begin{tabular}{lcc}
\hline Chile & 31 & 22 \\
Ecuador & 12 & 2 \\
Paraguay & 6 & 1 \\
Perú & 15 & 7 \\
Uruguay & 6 & 3 \\
Total & 126 & 65 \\
\hline
\end{tabular}

Fuente: Elaboración propia

En análisis estadísticos de los datos fueron expresados por medio de la estadística descriptiva, validación del instrumento por el método de análisis de consistencia interna de Alfa de Cronbach. Posteriormente se realizó el análisis factorial exploratorio para estudiar las relaciones de las dimensiones correspondientes, las pruebas t para muestras independientes, análisis de varianza (ANOVA), función discriminante y regresión lineal múltiple utilizando el programa estadístico statistical Product and Service Solutions (SPSS) en su versión 22.

\section{Resultados}

El primer análisis de la muestra está basado en la comparación de medias por las dimensiones correspondientes a las variables de factores ambientales externos, factores organizativos internos y mecanismos de apoyo al emprendimiento con relación a la naturaleza de las universidades, específicamente en lo relacionado al tipo de dependencia de la institución (estatal/privada), disposición de un parque tecnológico y al país de origen y de forma separada la dimensión de resultados como universidad emprendedora. Lo anterior debido a que las primeras tres variables corresponden a variables independientes y la cuarta es la variable dependiente.

Los resultados mostrados por el Gráfico 1 evidencian las diferencia entre las dimensiones de las variables factores ambientales externos y factores organizativos internos, con medias de 3.10 y 3.23 para las dimensiones de la primera 
variable y de 3.52 para apoyo administrativo, 3.30 para misión y estrategia y 3.26 para diseño organizacional y cuyos valores de prueba $t$ se ven reflejados en la tabla 5.
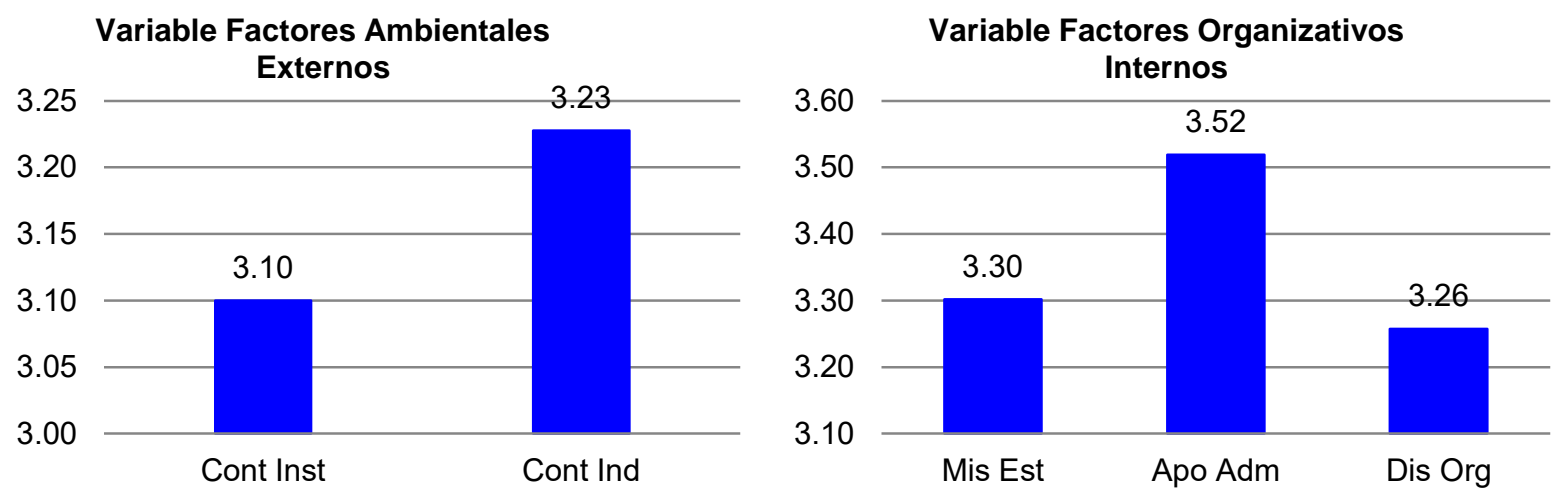

Gráfico 1. Resultados de las medias para las dimensiones de las variables Factores Ambientales Externos y Factores Organizativos Internos

Tabla 5

Prueba t para variables factores ambientales externos

Prueba para una muestra: Valor de prueba $=14.27$

\begin{tabular}{|c|c|c|c|c|c|}
\hline$t$ & gl & $\begin{array}{l}\text { Sig. } \\
\text { (bilat }\end{array}$ & $\begin{array}{l}\text { Diferen } \\
\text { cia de }\end{array}$ & $\begin{array}{l}95 \% \text { In } \\
\text { confial }\end{array}$ & rvalo de \\
\hline & & eral) & medias & $\begin{array}{l}\text { Inferi } \\
\text { or }\end{array}$ & Superior \\
\hline $\begin{array}{l}- \\
3,73 \\
0\end{array}$ & 64 &, 000 & $-1,87$ & $\begin{array}{l}- \\
2,871\end{array}$ &,- 868 \\
\hline $\begin{array}{l}3,20 \\
3\end{array}$ & 64 &, 002 & 1,86 &, 7030 & 3,033 \\
\hline
\end{tabular}

Prueba t para variables factores organizativos internos

Prueba para una muestra: Valor de prueba $=12.30$

\begin{tabular}{llllll}
$t$ & gl & $\begin{array}{l}\text { Sig. } \\
\text { (bilate } \\
\text { ral) }\end{array}$ & $\begin{array}{l}\text { Diferen } \\
\text { medias de }\end{array}$ & $\begin{array}{l}\text { 95\% Intervalo de } \\
\text { confianza } \\
\text { Inferior }\end{array}$ & $\begin{array}{l}\text { Superio } \\
\text { r }\end{array}$ \\
\hline $\begin{array}{l}1,42 \\
6\end{array}$ & 64 &, 159 &, 80769 &,- 3237 & 1,9391 \\
- & 64 &, 000 & - & $-2,2847$ &,- 7460 \\
3 & & & 1,51538 & &
\end{tabular}


Revista de Investigación Universitaria | Volumen 6 - Número 2, Julio - Diciembre, 2017 |

DOI :

$(\mathrm{cc}) \mathrm{BY}$

$$
\begin{array}{llllll}
\text { Dis_Org } & 1,68 \quad 64 \quad, 096 \quad, 73077 \quad-, 1342 & 1,5958 \\
8 & & &
\end{array}
$$

Aun cuando existe diferencia significativa para el resultado en las dos dimensiones de la variable factores ambientales externos $(\mathrm{p}<0.05)$, solamente la dimensión apoyo administrativo tiene diferencia significativa dentro de su variable factores organizativos internos $(\mathrm{p}<0,05)$ lo que demuestra que en ambas variables existe mayor énfasis en el contexto industrial y en la gestión administrativa que en el resto de las dimensiones.

El problema del diseño organizacional no afecta de igual forma a todas las instituciones de educación superior y las limitaciones son principalmente de participación, la conformación de estructuras jerárquicas y liderazgos internos (Silva, 2006). No debemos desconocer que el crecimiento de instituciones de educación superior trae aparejado el funcionamiento de sus propios postulados, lo que no siempre va acompañado del aseguramiento de la calidad (Gregorutti, Pavoni \&; Ramirez, 2014), pero por sobre estos considerandos, el diseño debe estar comprometido con los estándares de calidad en la gestión y efectividad en los propósitos que declara.

Los resultados de la variable mecanismos de apoyo al emprendimiento mostraron que las universidades apoyan con bajos fondos para el emprendimiento (Gráfico 2), para el desarrollo del emprendimiento en el personal y tienen baja educación para el emprendimiento. Estos resultados se consideran bajos, lo que demuestra que las instituciones debieran incrementar los recursos para el emprendimiento dentro de la universidad (a nivel de sus estudiantes y docentes) y su compromiso con el desarrollo académico empresarial. 
Según Clark, (2004a), una de las características que identifican a las EU es la capacidad de motivación del cuerpo académico/personal y la estimulación con incentivos económicos que promuevan el emprendimiento, por lo que indicadores bajos en esta dimensión podrían sugerir que aún es necesario consolidar el emprendimiento universitario para que generen una cultura natural de emprendimiento. Esto significa que las UE estarán involucradas en asociaciones, redes y múltiples relaciones con organizaciones públicas y privadas de tal forma que éstas sean un paraguas protector de interacción y cooperación (Inzelt, 2004), pero también este tipo de institución adoptan nuevas estructuras organizativas y políticas de incentivos para sus estudiantes y personal tales como un curriculum especializado, cursos de emprendimiento, incorporación de emprendedores a los programas académicos, entre otros (Tijssen, 2006).

\section{Variable Factores Mecanismos de Apoyo al Emprendimiento}

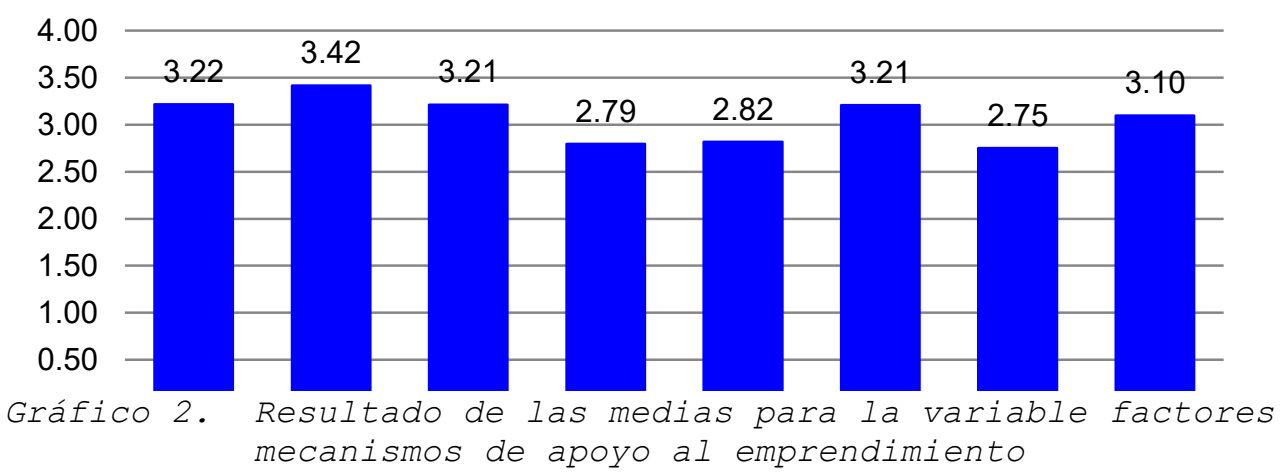

Los resultados estadísticos mostraron diferencias significativas para todas las dimensiones de la variable lo que significa que las universidades que ponen mayor énfasis en el intercambio de docentes y estudiantes (3.42) como factor de emprendimiento, las que tienen mayor desarrollo del emprendimiento educativo (3.21), apoyo universitario para el emprendimiento (3.21), así como la generación de nuevos 
negocios del personal que son apoyados por las políticas de la institución (3.22), serán instituciones más emprendedoras que aquellas que no han desarrollado estas dimensiones, cuyos valores de prueba $t$ se ven reflejados en la Tabla 6 .

Tabla 6

Prueba t para variable mecanismos de apoyo al emprendimiento

Prueba para una muestra: Valor de prueba $=10.71$

\begin{tabular}{|c|c|c|c|c|c|c|}
\hline & t & gl & $\begin{array}{l}\text { Sig. } \\
\text { (bilat } \\
\text { eral) }\end{array}$ & $\begin{array}{l}\text { Diferen } \\
\text { cia de } \\
\text { medias }\end{array}$ & $\begin{array}{l}\text { 95\% Inter } \\
\text { confianza } \\
\text { Inferio } \\
\text { r }\end{array}$ & $\begin{array}{l}\text { alo de } \\
\text { Superior }\end{array}$ \\
\hline Reg_Pol & $\begin{array}{l}- \\
2,36 \\
9\end{array}$ & 64 &, 021 & $\begin{array}{l}- \\
1,0638 \\
5\end{array}$ & $-1,9609$ &,- 1668 \\
\hline Inter & $\begin{array}{l}5,76 \\
5\end{array}$ & 64 &, 000 & $\begin{array}{l}2,9515 \\
4\end{array}$ & 1,9287 & 3,9744 \\
\hline AUPA & $\begin{array}{l}3,73 \\
3\end{array}$ & 64 &, 000 & $\begin{array}{l}2,1361 \\
5\end{array}$ &, 9929 & 3,2794 \\
\hline Ind_Curr & $\begin{array}{l}5,04 \\
2\end{array}$ & 64 &, 000 & $\begin{array}{l}3,2592 \\
3\end{array}$ & 1,9680 & 4,5505 \\
\hline $\begin{array}{l}\text { Fond_Emp } \\
r\end{array}$ & $\begin{array}{l}- \\
5,23 \\
5\end{array}$ & 64 &, 000 & $\begin{array}{l}- \\
2,2638 \\
5\end{array}$ & $-3,1278$ & $-1,3999$ \\
\hline Emp_Educ & $\begin{array}{l}- \\
2,41 \\
4\end{array}$ & 64 &, 019 & $\begin{array}{l}- \\
1,0946 \\
2\end{array}$ & $-2,0005$ &,- 1887 \\
\hline $\begin{array}{l}\text { Empr_- } \\
\text { Pers }\end{array}$ & $\begin{array}{l}- \\
5,74 \\
9\end{array}$ & 64 &, 000 & $\begin{array}{l}- \\
2,4638 \\
5\end{array}$ & $-3,3200$ & $-1,6077$ \\
\hline Met_Ensa & $\begin{array}{l}- \\
3,16 \\
4\end{array}$ & 64 &, 002 & $\begin{array}{l}- \\
1,4176 \\
9\end{array}$ & $-2,3130$ &,- 5224 \\
\hline
\end{tabular}

Estos resultados son concordantes con los estudios de Sullivan, (2011) sobre internacionalización de estudiantes y docentes en universidades a nivel mundial y que pone el énfasis en la planificación de la internacionalización por parte de 
dichas instituciones y posiciona a Sudamérica como el continente con el mal alto nivel de planificación en esta dimensión. De igual forma Rothaermel et al., (2007) afirma que cuando se apoya e incentiva el emprendimiento por parte de las universidades, se puede transformar el conocimiento que poseen en innovaciones que generarán nuevas organizaciones, lo que focaliza a las universidades como influyentes en la formación de futuros emprendedores y la coloca como bisagra entre la industria y el gobierno (Hussain, Bhuiyan, Said, \& Ab, 2017).

Por otro lado, los resultados de las dimensiones de la variable dependiente llamada "Resultados de Universidad Emprendedora" (ver Gráfico 3), muestran valores bajos para movilidad hacia la industria (2.78), Spin-off estudiantil (2.69), Spin-off Académico (2.41), patente y licenciamiento (2.58) Por otro lado de las siete dimensiones que conforman la variable, seis obtuvieron diferencia estadística significativa con la sola excepción de la dimensión "red" ( $\mathrm{p}>0.05$ ) según lo muestra el Gráfico 3.

\section{Variable Factores Resultado Universidad Emprendedora}

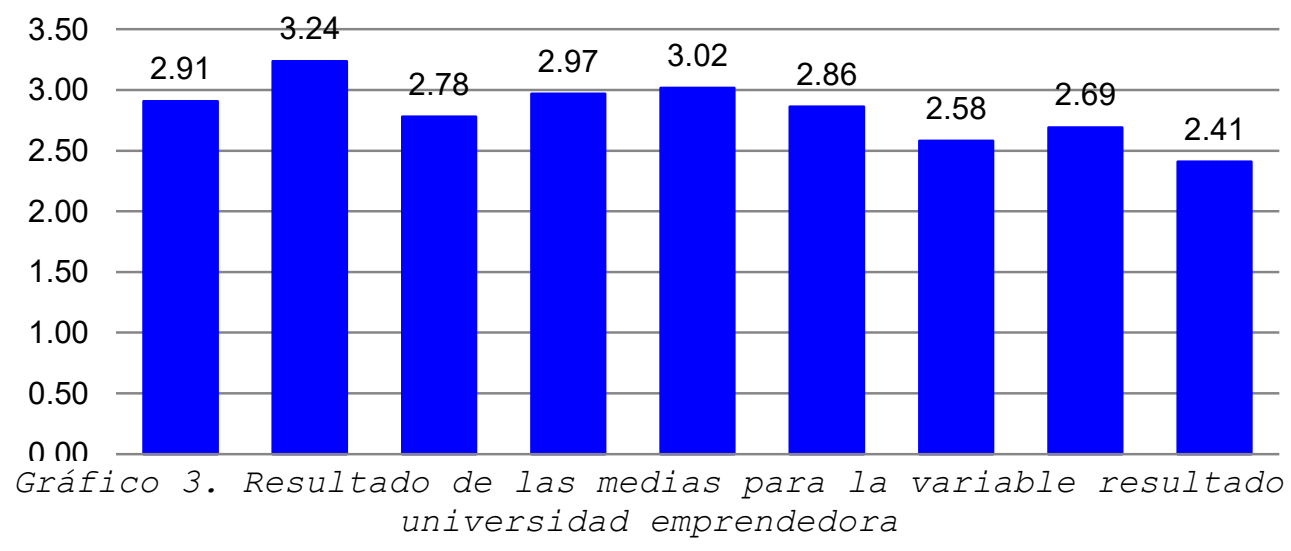

Tabla 7

Prueba t para variable resultado de universidad emprendedora

Prueba para una muestra: Valor de prueba $=9.41$

t $\quad g 1$

95\% Intervalo de confianza 


\begin{tabular}{|c|c|c|c|c|c|c|}
\hline & & & $\begin{array}{l}\text { Sig. } \\
\text { (bilate } \\
\text { ral) }\end{array}$ & $\begin{array}{l}\text { Diferenc } \\
\text { ia de } \\
\text { medias }\end{array}$ & Inferior & Superior \\
\hline $\begin{array}{l}\mathrm{Div} \_\mathrm{Co} \\
\mathrm{n}\end{array}$ & $\begin{array}{l}- \\
2,00 \\
4\end{array}$ & 64 &, 049 &,- 68692 & $-1,3719$ &,- 0020 \\
\hline Red &, 868 & 64 &, 389 &, 29769 &,- 3875 &, 9829 \\
\hline $\begin{array}{l}\text { Mov_In } \\
\mathrm{d}\end{array}$ & $\begin{array}{l}- \\
2,77 \\
0\end{array}$ & 64 &, 007 & $-1,07154$ & $-1,8444$ &,- 2987 \\
\hline $\begin{array}{l}\mathrm{Col}_{\mathrm{V}} \mathrm{In} \\
\end{array}$ & $\begin{array}{l}- \\
2,03 \\
6\end{array}$ & 64 &, 046 &,- 82538 & $-1,6353$ &,- 0155 \\
\hline $\begin{array}{l}\text { Pat_Li } \\
\text { C }\end{array}$ & $\begin{array}{l}- \\
3,50 \\
8\end{array}$ & 64 &, 001 & $-1,67154$ & $-2,6235$ &,- 7196 \\
\hline Sp_Est & $\begin{array}{l}3,02 \\
9\end{array}$ & 64 &, 004 & 1,35923 &, 4629 & 2,2556 \\
\hline $\mathrm{SP} \_\mathrm{Aca}$ & $\begin{array}{l}4,78 \\
1\end{array}$ & 64 &, 000 & 2,63615 & 1,5346 & 3,7377 \\
\hline
\end{tabular}

Llama la atención de los bajos resultados de la dimensión de patente y licenciamiento ya que se espera que el trabajo de emprendimiento de una institución termine en la venta/arriendo de licencias y patentes. Según el informe de (Reyes-Gatica \& Ripamonti, 2008) el proceso de licenciamiento y patentamiento dura en promedio 5 años, con múltiples requisitos que cumplir y con una muy alta competencia de patentamiento de parte de empresas extranjeras trabajando dentro de los países lo que genera un muy alto porcentaje de solicitudes abandonadas, principalmente debido a que no logran dar respuesta a tiempo de las observaciones hechas por los entes correspondientes. Esto podría ser una posible explicación que interprete los bajos resultados obtenidos en esta dimensión.

Por otro lado, los bajos niveles de spin-off académico y estudiantil pudieran tener su interpretación en el hecho que 
fuera de Europa y Estados Unidos, en Latinoamérica no se desarrollan de forma exitosa (Visintin \& Pittino, 2014) lo que ha impulsado a las universidades de investigación a establecer oficinas de transferencia tecnológica, sin embargo el desafío pendiente es que al no existir una regulación sobre este tipo de empresas de spin-off normalmente ocasiona conflicto de interés entre los académicos e investigadores con sus propias casas de estudio (Dias \& Silva-Porto, 2014).

Estas limitaciones legislativas pueden ser un desincentivo para la creación de empresas tipo spin-off (Bacchiocchi \& Montobbio, 2009). De igual forma los datos también nos muestras que el mayor efecto como característica de universidad emprendedora se ve reflejado por los nexos y conexiones institucionales y la educación continua de sus egresados y profesionales y los elementos asociados a la generación de consultorías, colaboración con la investigación, divulgación del conocimiento y movimiento hacia la industria.

Con relación al contraste de las hipótesis, el estudio consideró los niveles de comparación para la estimación del coeficiente de determinación de los resultados los sugeridos por la literatura (Henseler, Ringle \& Sinkovics, 2009; Hair, Ringle \& Sarstedt, 2011) y que están reflejados en la Tabla.

Tabla 8

Categorías para nivel de coeficiente de determinación

\begin{tabular}{lccc}
\hline \multicolumn{4}{c}{ Coeficiente de determinación $\mathbf{R}^{\mathbf{2}}$} \\
\hline & Débil & Moderado & Sustancial \\
Hair, (2011) & $<0,25$ & 0,5 & 0,75 \\
$\begin{array}{l}\text { Henseler et al., } \\
(2009)\end{array}$ & $<0,19$ & 0,33 & 0,67 \\
\hline
\end{tabular}

La primera hipótesis señalaba que "los factores de desarrollo externo influencian positiva y significativamente en los resultados del modelo de gestión en una institución 
tipificada como Universidad Emprendedora". Ante ella, los resultados de correlación que vinculan ambas variables muestran un valor para la variable dependiente según la siguiente fórmula: $y=3 x+(-10)$ tal cual lo muestra el Gráfico 4.

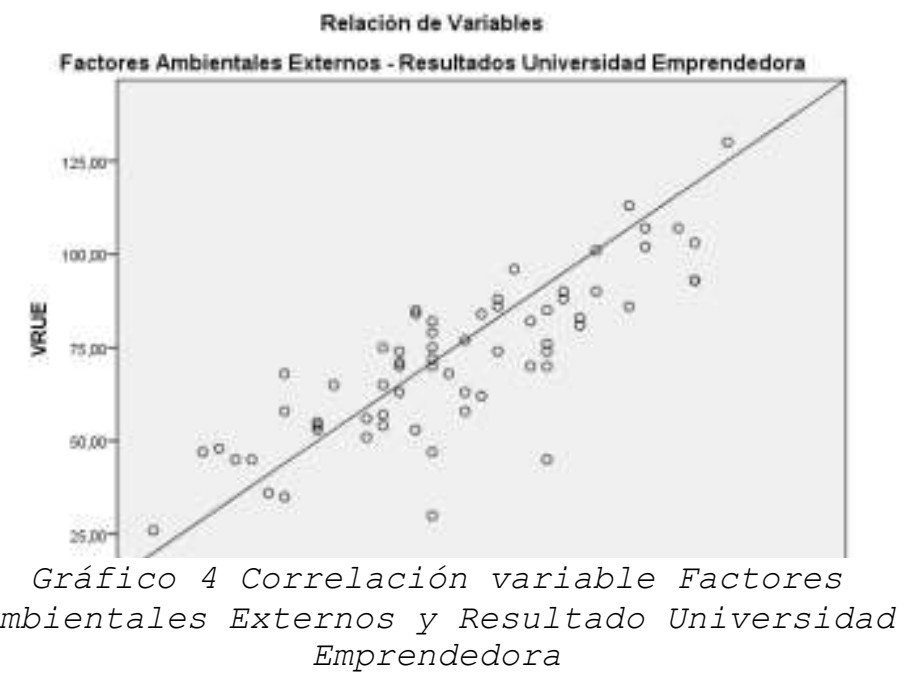

El análisis del Gráfico 4 es complementada con los datos entregados en la Tabla que nos entrega el valor de la correlación correspondiente a $r=0.821$ con un valor $\mathrm{p}=0.000$ y un $r^{2}=0.673$. Esto significa que el 82,1\% de los datos se relacionan entre sí, es decir, que los resultados obtenidos a nivel de los factores ambientales externos se relacionan positiva y significativamente con los resultados obtenidos en la universidad que tienen el modelo de gestión de una universidad emprendedora y el valor de $r^{2}$ señala que el 67,3\% de la variación en Y es explicada por la recta de la regresión. Estos resultados nos permiten confirmar la primera hipótesis específica de la investigación.

Tabla 9

Resultados regresión de las variables factores ambientales externos y resultado universidad emprendedora

\section{Resumen del modelo}


Revista de Investigación Universitaria | Volumen 6 - Número 2, Julio - Diciembre, 2017 | DOI :

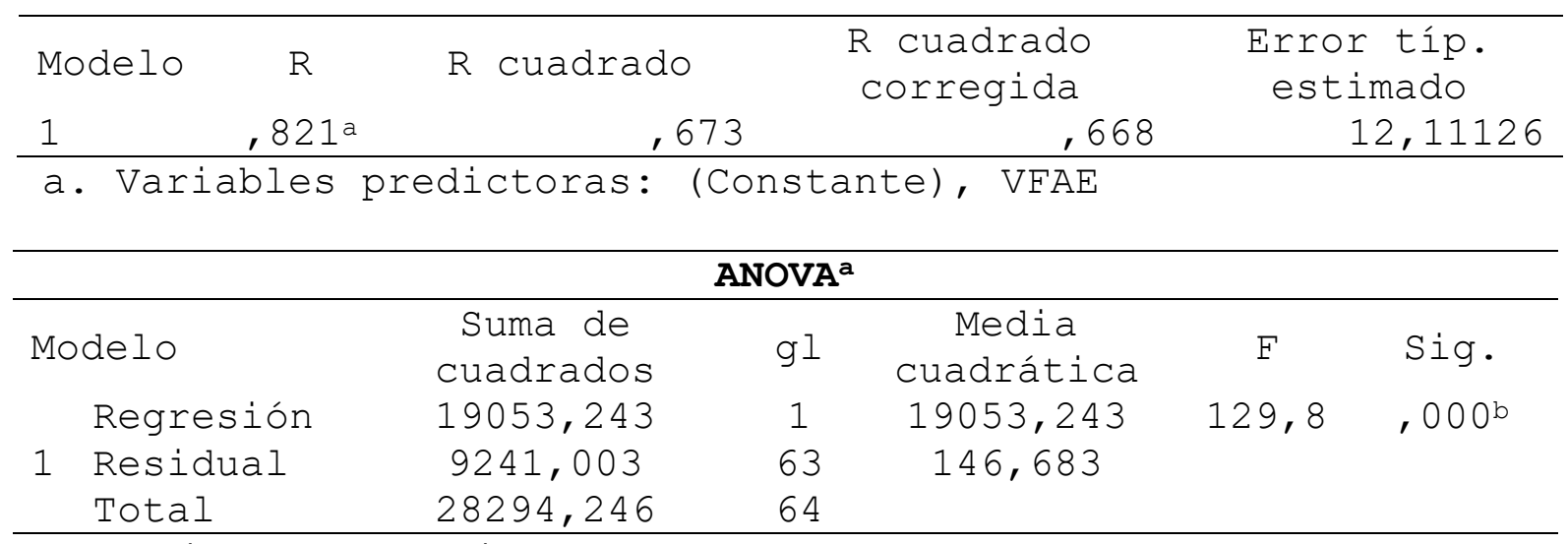

a. Variable dependiente: VRUE

b. Variables predictoras: (Constante), VFAE

\begin{tabular}{|c|c|c|c|c|c|}
\hline \multicolumn{6}{|c|}{ Coeficientes $^{a}$} \\
\hline \multirow{2}{*}{ Modelo } & $\begin{array}{l}\text { Coefic } \\
\text { estand }\end{array}$ & $\begin{array}{l}\text { s no } \\
\text { ados }\end{array}$ & $\begin{array}{c}\text { Coeficientes } \\
\text { tipificados }\end{array}$ & \multirow{2}{*}{ t } & \multirow{2}{*}{ Sig. } \\
\hline & B & $\begin{array}{l}\text { Error } \\
\text { típ. }\end{array}$ & Beta & & \\
\hline \multirow[t]{2}{*}{$\begin{array}{l}\text { (Constan } \\
1 \text { te) }\end{array}$} & 11,339 & 5,521 & & 2,054 &, 044 \\
\hline & 2,122 &, 186 &, 821 & $\begin{array}{c}11,39 \\
7\end{array}$ &, 000 \\
\hline
\end{tabular}

La segunda hipótesis señalaba que "los factores de desarrollo interno influencian positiva y significativamente en los resultados del modelo de gestión en una institución tipificada como Universidad Emprendedora", los resultados de correlación que vinculan ambas variables muestran un valor para la variable dependiente según la siguiente fórmula: y=2.4x + (4) tal cual lo muestra el Gráfico 5 . 


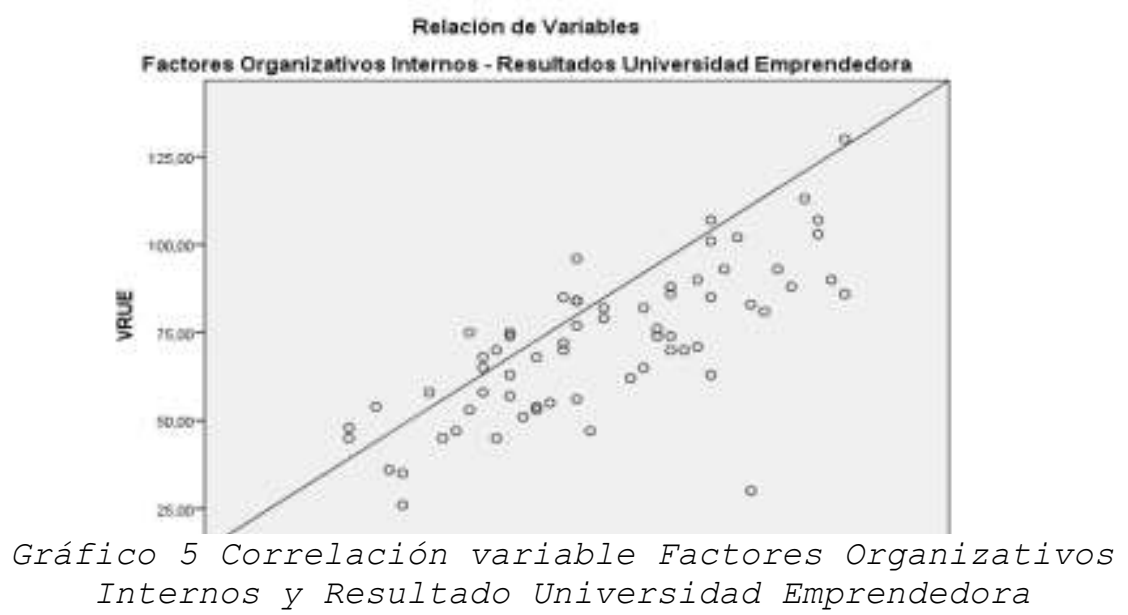

La tabla 10 complementa los resultados entregados en el Gráfico 5 para su análisis señalando un valor de la correlación correspondiente a $r=0.736$ con un valor $p=0.000$ y un $r^{2}=0.542$. Esto significa que el $73,6 \%$ de los datos se relacionan entre sí, es decir, que los resultados obtenidos a nivel de los factores organizativos interno se relacionan positiva y significativamente con los resultados obtenidos en la universidad que tienen el modelo de gestión de una universidad emprendedora y el valor de $r^{2}$ señala que el 54,2\% de la variación en $Y$ es explicada por la recta de la regresión, confirmando la segunda hipótesis.

Tabla 10

Resultados regresión de las variables factores organizativos internos, y resultado universidad emprendedora

\section{Resumen del modelo}

\begin{tabular}{|c|c|c|c|c|}
\hline Modelo & & R cuadrado & $\begin{array}{l}\text { R cuadrado } \\
\text { corregida }\end{array}$ & $\begin{array}{l}\text { Error típ. de la } \\
\text { estimación }\end{array}$ \\
\hline 1 & $.736 a$ &, 542 &, 535 & 14,343 \\
\hline
\end{tabular}

\section{ANOVA $^{a}$}

\begin{tabular}{|c|c|c|c|c|c|}
\hline Modelo & $\begin{array}{l}\text { Suma de } \\
\text { cuadrados }\end{array}$ & gl & $\begin{array}{c}\text { Media } \\
\text { cuadrática }\end{array}$ & F & Sig. \\
\hline Regresión & 15332,70 & 1 & 15332,70 & 74,525 &, $000^{b}$ \\
\hline$\perp$ Residual & 12961,55 & 63 & 205,74 & & \\
\hline
\end{tabular}


Revista de Investigación Universitaria | Volumen 6 - Número 2, Julio - Diciembre, 2017 | DOI :

Total

28294,25

64

a. Variable dependiente: VRUE

b. Variables predictoras: (Constante), VFOI

Coeficientes ${ }^{a}$

\begin{tabular}{|c|c|c|c|c|c|}
\hline \multirow[t]{2}{*}{ Modelo } & \multicolumn{2}{|c|}{$\begin{array}{c}\text { Coeficientes no } \\
\text { estandarizados }\end{array}$} & \multirow{2}{*}{$\begin{array}{c}\text { Coeficientes } \\
\text { tipificados } \\
\text { Beta }\end{array}$} & \multirow[t]{2}{*}{$t$} & \multirow[t]{2}{*}{ Sig. } \\
\hline & B & Error típ. & & & \\
\hline (Constante) & 14,026 & 6,935 & & 2,02 &, 047 \\
\hline VMAE & 1,567 &, 182 &, 736 & 8,63 &, 000 \\
\hline
\end{tabular}

a. Variable dependiente: VRUE

Finalmente, la tercera hipótesis señalaba que "los mecanismos de apoyo al emprendimiento influencian positiva y significativamente en los resultados del modelo de gestión en una institución tipificada como Universidad Emprendedora", los resultados de correlación que vinculan ambas variables muestran un valor para la variable dependiente según la siguiente fórmula: $y=1,2 x+(28)$ tal cual lo muestra la tabla 10.

Relación de Variables

Mecanismos Apoyo Emprendimiento - Resultados Universidad Emprendedora

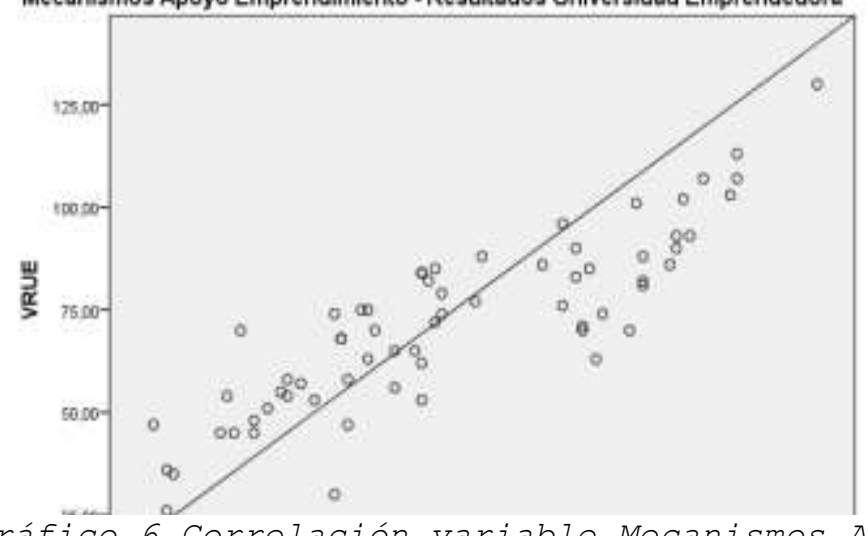

Gráfico 6 Correlación variable Mecanismos Apoyo Emprendimiento y Resultado Universidad Emprendedora

VMAE

La

tabla 11

complementa los resultados entregados en el gráfico 6 para su análisis señalando un valor de la correlación correspondiente a $r=0.863$ con un valor $p=0.000$ y un $r^{2}=0.745$. Esto significa 
Revista de Investigación Universitaria | Volumen 6 - Número 2, Julio - Diciembre, 2017 | $\mathrm{DOI}:$

que el 86,3\% de los datos se relacionan entre sí, es decir, que los resultados obtenidos a nivel de los factores organizativos interno se relacionan positiva y significativamente con los resultados obtenidos en la universidad que tienen el modelo de gestión de una universidad emprendedora y el valor de $r^{2}$ señala que el 74,5\% de la variación en y es explicada por la recta de la regresión. Estos resultados nos permiten confirmar la tercera hipótesis específica de la investigación.

Tabla 11

Resultados regresión de las variables mecanismos apoyo emprendimiento, y resultado universidad emprendedora

\section{Resumen del modelo}

\begin{tabular}{|c|c|c|c|c|}
\hline Modelo & $\mathrm{R}$ & R cuadrado & $\begin{array}{l}\text { R cuadrado } \\
\text { corregida }\end{array}$ & $\begin{array}{l}\text { Error típ. de la } \\
\text { estimación }\end{array}$ \\
\hline 1 &, $863^{a}$ &, 745 &, 741 & 10,69774 \\
\hline
\end{tabular}

\section{ANOVA $^{a}$}

\begin{tabular}{|c|c|c|c|c|c|c|}
\hline \multicolumn{2}{|c|}{ Modelo } & $\begin{array}{c}\text { Suma de } \\
\text { cuadrados }\end{array}$ & gl & $\begin{array}{c}\text { Media } \\
\text { cuadrática }\end{array}$ & F & Sig. \\
\hline \multirow{3}{*}{1} & Regresión & 21084,421 & 1 & 21084,421 & 184,237 &, $000^{b}$ \\
\hline & Residual & 7209,825 & 63 & 114,442 & & \\
\hline & Total & 28294,246 & 64 & & & \\
\hline
\end{tabular}

a. Variable dependiente: VRUE

b. Variables predictoras: (Constante), VMAE

\begin{tabular}{|c|c|c|c|c|c|}
\hline \multicolumn{6}{|c|}{ Coeficientes $^{a}$} \\
\hline \multirow{3}{*}{$\begin{array}{l}\text { Modelo } \\
\qquad \begin{array}{l}\text { (Consta } \\
1 \text { nte) }\end{array}\end{array}$} & \multicolumn{2}{|c|}{$\begin{array}{l}\text { Coeficientes no } \\
\text { estandarizados }\end{array}$} & \multirow{2}{*}{$\begin{array}{c}\text { Coeficientes } \\
\text { tipificados } \\
\text { Beta }\end{array}$} & \multirow[t]{2}{*}{$t$} & \multirow{2}{*}{$\begin{array}{c}\text { Sig } \\
\text {. }\end{array}$} \\
\hline & B & Error típ. & & & \\
\hline & $\begin{array}{c}11,47 \\
6\end{array}$ & 4,645 & & 2,47 & $\begin{array}{l}, 01 \\
6\end{array}$ \\
\hline VMAE &, 705 &, 052 &, 863 & $\begin{array}{c}13,5 \\
7\end{array}$ & $\begin{array}{l}00 \\
0\end{array}$ \\
\hline
\end{tabular}

\section{Resumen y conclusiones}

Con el propósito de confirmar las tres hipótesis de investigación para el logro de los objetivos se analizó el 
Revista de Investigación Universitaria | Volumen 6 - Número 2, Julio - Diciembre, 2017 | DOI :

modelo de forma empírica con la realidad sudamericana de los 8 países participantes del estudio. El análisis de regresión permitió confirmar dichas hipótesis.

Durante el desarrollo de la investigación se pudo corroborar que muchas instituciones de manera intuitiva realizan acciones que la literatura comienza a definir como una característica emprendedora institucional. Esto comienza a materializarse con más rapidez en las universidades privadas que en las instituciones públicas ya que éstas últimas tienen modelos de gestión más rígidos cuya dependencia hace que las adaptaciones a las necesidades del entorno sean más lentas; sin embargo, tienen el financiamiento del estado para su desarrollo.

Aun cuando no estaba dentro de los objetivos de esta investigación, nuevas pesquisas podrían hacer categorizaciones por países que pudieran cruzarse con factores político-sociales que promuevan o retrasen la gestión de instituciones emprendedoras. Igualmente, nuevas investigaciones podrían categorizar instituciones emprendedoras según las similitudes por subgrupos con el propósito de identificar nuevas razones de éxito-fracaso en los procesos de gestión que sean directamente vinculantes al modelo estudiado.

De igual forma, si bien el estudio tuvo una alta participación de las universidades que constituían el universo, es necesario hacer mayores esfuerzos para lograr la participación de más instituciones de algunos países cuyos representantes constituyeron una minoría con relación a la muestra total y el desafío podría incluir no solo a Sudamérica sino considerar como universo a todos los países de Latinoamérica.

\section{Referencias}


Revista de Investigación Universitaria | Volumen 6 - Número 2, Julio - Diciembre, 2017 | DOI :

Altmann, A; Ebersberger, B. (2013). Universities in change: A brief introduction. In Managing Higher Education Institutions in the Age of Globalization. (B. Altmann, A; Eberberger, Ed.). New York, NY: Springer.

Amo, B. (2006). The influence from corporate entrepreneurship and intrapreneurship on white-collar workers' employee innovation behaviour. International Journal of Innovation and Learning, 3(3), 284-298.

Audretsch, D. (2014). From the entrepreneurial university to the university for the entrepreneurial society. The Journal of Technology Transfer, 39(3), 313-321. http://doi.org/10.1007/s10961-012-9288-1

Bacchiocchi, F. \& Montobbio, F. (2009). Knowledge diffusion from university and public research. A comparison between US, Japan and Europe using patent citations. The Journal of Technology Transfer, 34(2), 169-181.

Belousova, O. Bailly, Benoit \& Basso, O. (2010). A conceptual model of corporate entrepreneurial behavior. Lousanne, Switzerland.

Benneworth, P. (2007). Seven Samurai Opening Up the Ivory Tower? The Construction of Newcastle as an Entrepreneurial University. European Planning Studies, 15(4), 487-509.

Bernasconi, A. (2013). The Profit Motive in Higher Education. International Higher Education, 71, 8-10.

Blenker, Per; Dreisler, Poul \& Kjeldsen, J. (2006). Entrepreneurship Education - The New Challenge Facing the Universities. Aarhus, Denmark: Pile-Group.

Bratianu, Constantin; Stanciu, S. (2010). An overview of present research related to entrepreneurial university. Management \& Marketting, 5(2), 117-134.

Cancino, Victor; Schmal, R. (2014). Sistema de Acreditacion Universitaria en Chile: ¿Cuánto hemos avanzado? Estudios Pedagógicos, XI(1), 41-60.

Cargill, B. (2007). Models of organisational and managerial capability for the entrepreneurial university in Australia. Swinburne University of Technology.

Chrisman, J. (1995). Faculty entrepreneurship and economic development: The case of the University of Calgary. Journal 
Revista de Investigación Universitaria | Volumen 6 - Número 2, Julio - Diciembre, 2017 | DOI :

of Business Venturing, 10(4), 267-281.

http://doi.org/doi.org/10.1016/0883-9026(95)00015-Z

Clark, B. (1998). Creating entrepreneurial universities: organizational pathways of transformation. Pergamon.

Clark, B. (2004). Sustaining Change in Universities, Society for Research into Higher Education. Londres: Open University Press.

CNDE . (2012). ACUERDO No 106/2012. Santiago.

CNDE. (2014). Estadisticas y bases de datos. Retrieved from http://www. cned.cl/public/secciones/SeccionIndicesPostulan tes/indices_pregrado.aspx

Covin, Ji Slevin, D. (1991). A conceptual model of entrepreneurship as firm behavior. Entrepreneurship Theory and Practice, (Autumn isseu), 7-25.

Crow, M. (2008). Bulding an entrepreneurial university. In Third Annual Kauffman Foundation-Max Planck Institute (p. 17). Munich.

Dias, A. \& Silva-Porto, G. (2014). Como a USP transfere tecnologia? Organizações \& Sociedade, 21(70), 489-507. http://doi.org/10.1590/S1984-92302014000300008

Dooley, L\& Kirk, D. (2007). University-industry collaboration: Grafting the entrepreneurial paradigm onto academic structures. European Journal of Innovation Management, $10(3), 316-332$.

Etzkowitz. (1983). Entrepreneurial scientists and entrepreneurial universities in American academic science. Minerva, 21(2), 198-233. http://doi.org/10.1007/BF01097964

Etzkowitz, H; Ranga, M. \& D. J. (2012). Whither the university? The Novum Trivium and the transition from industrial to knowledge society. Social Cience Information, 51(2), 143164. http://doi.org/10.1177/0539018412437099

Etzkowitz, H. (2003a). Innovation in innovation: the Triple Helix of university-industry-government relations. Social Science Information, 42, 293-337. http://doi.org/10.1177/05390184030423002

Etzkowitz, H. (2003b). Innovation in Innovation: The triple helix of university-Industry-government relations. Social Science Information, 42, 293-337. 
http://doi.org/10.1177/05390184030423002

Etzkowitz, H. (2004). The evolution of the entrepreneurial univesity. International Journal of Technology and Globalisaton, 1(1), 64-77.

Gajon, E. (2011). Internal Factors Affecting the Entrepreneurial University: the Case of the Tecnológico de Monterrey (Mexico). Barcelona.

Gibb, A \& Hannon, P. (2005). Towards the entrepreneurial university. Journal of Entrepreneurship Education, 4(1), $73-110$.

Gray, D. (2014). Doing Research in the Real Worl. (J. Seaman, Ed.) (threeth). London: SAGE Publications Ltd.

Gregorutti, Gustavo; Pavoni, Caterina; Ramirez, N. (2014). Las acreditaciones y sui impacto en universidades adventistas de latinoamérica. Revista Apunte Universitario, IV(2), 924 .

Grimaldi, R; Kenney, Martin; Siegel, Donald; Wright, M. (2011). 30 years after Bayh-Dole: Reassessing academic entrepreneurship. Research Policy 40, 1045-1057. http://doi.org/10.1016/j.respol.2011.04.005

Guenther, Jutta \& Wagner, K. (2008). Getting out of the ivory tower - new perspectives on the entrepreneurial university. European Journan International Management, 2(4), 400-417.

Guerrero, M; Urbano, D. (2010). The creation and development of entrepreneurial universities in Spain: An institutional approach. Nova Science.

Guerrero, Maribel; Urbano, David; Kirby, D. (2006). A literature review on entrepreneurial universities: An institutional approach (p. 37). Barcelona: Departament d'Economía de l'Empresa.

Guerrero, Maribel; Urbano, D. (2012). Transferencia de conocimiento y tecnologia: Mejores practicas en las universidades emprendedoras españolas. Gestion Y Política Pública, XXI(1), 107-139.

Guerrero, M., Urbano, D., Cunningham, J., \& Organ, D. (2014). Entrepreneurial universities in two European regions: A case study comparison. Journal of Technology Transfer, 39(3) . http://doi.org/10.1007/s10961-012-9287-2 
Hair, J.; Ringle, Ch. \& Sarstedt, M. (2011). PLS-SEM: Indeed a silver Bullet. Journal of Marketing Theory and Practice, $19(2), 139-151$.

Henseler, J., Ringle, Ch, \& Sinkovics, R. (2009). The use of partial least squares path modeling in international marketing. In P. Sinkovics, R. \& Ghauri (Ed.), New Challenges to International Marketin (p. 277). Emerald Group Publishing Limited.

Henseler, J., Ringle, C. M., \& Sinkovics, R. R. (Eds.). (2009). Advances in International Marketing (Vol. 20). Bingley: Emerald Group Publishing. http://doi.org/10.1108/S14747979 (2009) 0000020014

Huggins, Robert; Johnston, A. (2009). The economic and innovation contribution of universities: a regional perspective. Environment and Planning $C$ : Government and Policy, 27(6), 1088-1106.

Hussain, M.; Bhuiyan, A.; Said, J. \& Ab, M. S. B. (2017). Entrepreneurship Education is the Key Contrivance of Poverty Alleviation: An Empirical Review. MAYFEB Journal of Business and Management, 1, 32-41.

Inzelt, A. (2004). The evolution of university-industrygovernment relationships during transition. Rresearch Policy, 33, 975-995.

Kirby, D. (2006). Creating Entrepreneurial Universities in the UK: Applying Entrepreneurship Theory to Practice. Journal of Technology Transfer, 31, 599-603.

Klofsten, Magnus; Jones-Evans, D. (2000). Comparing Academic Entrepreneurship in Europe - The Case of Sweden and Ireland. Small Business Economics, 14, 299-309.

Lolas, F. (2006). Sobre modelos de gestion universitaria. Calidad En La Educacion, 24, 37-45.

Markuerkiaga-Arritola, L. (2014). An Empirical-Institutional Analysis of Factors Affecting the Entrepreneurial University. Mondragon Unibertsitatea.

Markuerkiaga, Leire; Errasti, Nekane \& Igartua, J. (2014). Success factors for managing an entrepreneurial university. Industry \& Higher Education, 28(4), 233-244. http://doi.org/10.5367/ihe.2014.0214 
Mavi, R. (2014). Indicators of Entrepreneurial University: Fuzzy AHP and Fuzzy TOPSIS Approach. Journal of the Knowledge Economy, 5(2), 370-387.

Meyer, G. (2011). The Reinvention of Academic Entrepreneurship. Journal of Small Business Management, 49(1), 1-8.

Moreno-Brid, Juan Carlos; Ruiz-Nápoles, P. (2008). Public research universities in latin america and their relation to economic development. Mexico City.

Mowery, David; Sampat, Bhaven; Ziedonis, A. (2002). Learning to Patent: Institutional Experience, Learning, and the Characteristics of U.S. Universityu Patents After the BayhDole Act, 1981-1992. Management Science, 48(1), 73-89. http://doi.org/10.1287/mnsc.48.1.73.14278

OCDE. (2013a). La Educacion Superior en Chile. In El Aseguramiento de la Calidad en la Educacion Superior en Chile (p. 104). Santiago.

OCDE. (2013b). Nivel de educacion alcanzados en los países de la OCDE. In Panorama de la educacion 2013: indicadores de la OCDE (p. 443). Barcelona: Santillana Educacion.

Peterka, S. (2007). Entrepreneurial university as the most important leverage in achieving knowledge-based society. In The Ninth International Conference: "Challenges of Europe: Growth and Competitivness - Reversing the Trends" (p. 23). Split, Coacia.

Peterka, S. (2011). Entrepreneurial university as the most important leverage in achieving knowledge-based society. In V. Reic; Zlatan; Simie (Ed.), Callanges of Europe: Growth and comlpetitiveness - reversing the trends (p. 908). Split, Croacia: Faculty of Economics, University of Split.

Pinto, H., Fernandez-Esquinas, M., \& Uyarra, E. (2013). Universities and Knowledge-Intensive Business Services (KIBS) as Sources of Knowledge for Innovative Firms in Peripheral Regions. Regional Studies, 3404 (February 2016). http://doi.org/10.1080/00343404.2013.857396

Powers, J; Macdougall, P. (2005). University start-up formation and technology licensing with firms that go public: a resource-based view of academic entrepreneurship. Journal of Business Venturing, 20(3), 291-311.

Reyes-Gatica, A. \& Ripamonti, M. (2008). Transferencia 
Tecnológica en Chile: Uso de patentes y Licenciamiento". Universidad de Chile.

Roessner, David; Bond, Jennifer; Okubo, Sumiye; Planting, M. (2013). The economic impact of licensed commercialized inventions originating in university research. Research Policy, 42, 23-34. http://doi.org/10.1016/j.respol.2012.04.015

Rolando, Rodrigo; Salamanca, Juan; Aliaga, M. (2010). Evolución Matrícula Educación Superior de Chile Periodo 1990 - 2009. Santiago.

Röpke, J. (1998). Innovation, academic knowledge creation and regional development in a globalized economy. The Entrepreneurial University. Marburg, Germany.

Rothaermel, F; Agung, S \& Jiang, L. (2007). University entrepreneurship: a taxonomy of the literature. Industrial and Corporate Change, 16(4), 691-791.

Sampieri, R.; Fernández, C.; Baptista, P. (2014). Selección de la Muestra. (A. Martínez, Ed.)Metodología de la Investigacion (3a Ed.). Ciudad de Mexico: McGraw Hill.

Sanchez, Carlos; Ríos, H. (2011). La economía del conocimiento como base del crecimiento económico en México. Revista Venezolana de Información, 8(2), 43-60.

Silva, C. (2006). Aseguramiento de la calidad en la gestión de instituciones de educación superior: expectativas y desafíos. Revista Calidad En La Educacion, 24, 119-132.

Steffensen, M; Rogers, E \& Speakman, K. (2000). Spin-offs from research centers at a research university. Journal of Business Venturing, 15(1), 93-111.

Sullivan, J. (2011). Global leadership in higher education administration: Perspectives on internationalization by university presidents, vice-presidents and deans. Retrieved from http://scholarcommons.usf.edu/etd/3370/

Thompson, A; Strickland, A.; Gamble, John; Peteraf, M. (2012). Administracion Estratégica: Conceptos y Casos. (K. EstradaArriagada, Ed.) (18 Ed.). Mexico D.F.: McGraw Hill.

Tijssen, R. (2006). Universities and industrially relevant science: Towards measurement models and indicators of entrepreneurial orientation. Research Policy, 35, 1569- 
Revista de Investigación Universitaria | Volumen 6 - Número 2, Julio - Diciembre, 2017 | DOI :

1585.

Tuunainen, J. (2005). Contesting a Hybrid Firm at a Traditional University. Social Studies of Science, 35(2), 173-210.

Unesco, O. R. de E. de la. (2014). América Latina y el Caribe: Revisión regional 2015 de la Educacón para Todos. Santiago.

Valera-loza, D. H. (2010). La Universidad Emprendedora: Nuevos retos en la formación superior. Innovacion \& Emprendimiento. Revista Latinoamericana de Ciencias Empresariales, 1, 9-36.

Visintin, F. \& Pittino, D. (2014). Founding team composition and early performance of university-Based spin-off companies. Technovation, 34, 31-43. 